Atmospheric physics

\title{
Interaction between whistlers and radiation belt electrons
}

from M.J. Rycroft

ANYONE using a radio receiver during a thunderstorm is familiar with the fact that lightning produces radio waves. What is not so well known is that some of these radio waves, those at audio frequencies of a few $\mathrm{kHz}$, get trapped in ducts aligned along the Earth's magnetic field and bounce back and forth ('hop') along the dipolar field lines. The dispersive nature of the medium through which the waves travel ensures that, in general, the higher frequency components travel faster than the lower. When picked up on a broad band receiver, the waves are heard as a succession of descending whistles (hence 'whistlers'), the rate of descent decreasing on successive hops. On page 740 of this issue Voss et al ${ }^{1}$. report the first satellite observations of another direct consequence of lightning flashes: the dumping of energetic electrons from the magnetosphere into the upper atmosphere $^{1}$. Such phenomena have geophysical and astrophysical relevance.

The van Allen radiation belts contain electrons and ions that are trapped by the Earth's magnetic field, accelerated to energies of some tens of $\mathrm{keV}$ by a variety of plasma physical processes which occur in the magnetosphere, preferentially on the night-side of the Earth, and associated with auroral substorms. Theoretical considerations of the loss of electrons from the belts have focussed on the cyclotron resonant interaction between whistlers, the frequencies of which are below both the plasma frequency and the cyclotron frequency, and energetic electrons travelling in the opposite direction along a geomagnetic flux tube. The condition for cyclotron resonance is that the electron 'feels' the wave Doppler-shifted up to its cyclotron frequency. In the equatorial plane, the phase velocity - and also the group velocity - of whistler mode waves propagating along a geomagnetic flux tube in a duct of slightly enhanced plasma density is a minimum. Thus, the equatorial plane is where cyclotron resonance occurs with electrons of the least energy.

The first observation of such a waveparticle interaction in the magnetosphere was serendipitous; during the flight of a Petrel rocket, carrying Geiger counters and launched from the Outer Hebrides, a transient increase in the intensity of energetic electrons having pitch angles between $60^{\circ}$ and $120^{\circ}$ was recorded (Rycroft, M.J. Planet. Space Sci. 21,239; 1973). The increase, by a factor of 20 above the quasisteady intensity observed throughout the remainder of the flight, occurred in 0.8 seconds and was simultaneous for both $>45 \mathrm{keV}$ and $>110 \mathrm{keV}$ electrons. About half a second later a two-hop whistler was recorded on the ground. During the period of enhanced electron intensity, the entire seconds, four-, six- and eight-hop whistlers were also received. From an analysis of the whistlers were ducted through the magnetosphere along the $\mathrm{L}=3.3 \pm 0.1$ field plane, $\mathrm{N}$, was found to be $330 \pm 10 \mathrm{~cm}^{-3}$, a value that is characteristic of conditions within the plasmapause.

It was suggested that the association of whistler and electron intensity was not coincidental but the result of a cyclotron resonant interaction between energetic electrons and whistler mode waves moving in opposite directions along the $\mathrm{L}=\mathbf{3} .3 \mathrm{flux}$ tube. For gyroresonance on this flux tube at the equator, the parallel component of energy of the electrons is $25 \mathrm{keV}$ at $3 \mathrm{kHz}$ in the whistler band, or $100 \mathrm{keV}$ at $1 \mathrm{kHz}$ below it. A possible explanation is that energetic electrons of a sufficiently anisotropic pitch-angle distribution-function and associated with electrons injected during an earlier auroral substorm become unstable, through cyclotron resonance instability, when they drift into the high density plasmasphere. The instability causing the burst of electron precipitation was believed to be triggered by the half-hop whistler propagating across the equator. The whistler was amplified during the interaction; it was later observed, above the duration of which was only about six their spectrogram, it was concluded that line. The electron density in the equatorial

background noise level, as a two-hop whistler.

Voss et al. have measured this waveparticle interaction by sophisticated solidstate instrumentation cooled to $150 \mathrm{~K}$ aboard the $S$ 81-1 satellite in a polar orbit at $230 \mathrm{~km}$ altitude. They show several examples of one-hop whistlers, observed on the ground in Antarctica, that are associated with bursts of precipitating electrons. The flux of precipitating electrons is observed to rise by a factor of up to 100 within 0.2 seconds. Because the cyclotron resonance interaction reduces the pitch angle of energetic electrons trapped in the magnetosphere, their mirror points descend below the satellite altitude. For cyclotron resonance in the equatorial plane of the $L=2.2 \pm 0.1$ flux tube, $\mathrm{N}$ is estimated to be $3200 \mathrm{~cm}^{-3}$; waves with a frequency of $4 \mathrm{kHz}$ resonate with $150 \mathrm{keV}$ electrons. Such energetic particles can penetrate the atmosphere down to a height of $80 \mathrm{~km}$, where they cause a significant amount of secondary ionization. This can affect the characteristics of very low frequency radio waves propagating in the Earth-ionosphere waveguide.

The overall efficiency of whistlers in causing the loss of the van Allen radiation belt electrons is still not known. However, with a globally-averaged rate of about 100 lightning discharges per second (Brookes, C.E.P. Geophys. Mem., London 24; 1925) there is considerable scope for further experimental studies of this wave-particle interaction. Such studies are also of relevance to other magnetospheres in the Universe, and to the loss of charged particles from plasma devices in any Earthbound laboratory that relies on magnetic mirrors for plasma containment.

M.J. Rycroft is in the NERC British Antarctic Survey, Cambridge CB3 OET, UK.

A CORE sample, obtained in the course of test-drilling from a North Sea oil rig belonging to Marathon Oil, produced this 150-million year old fossil of the front half of the fish Tharsis dubius, now in the British Museum of Natural History.

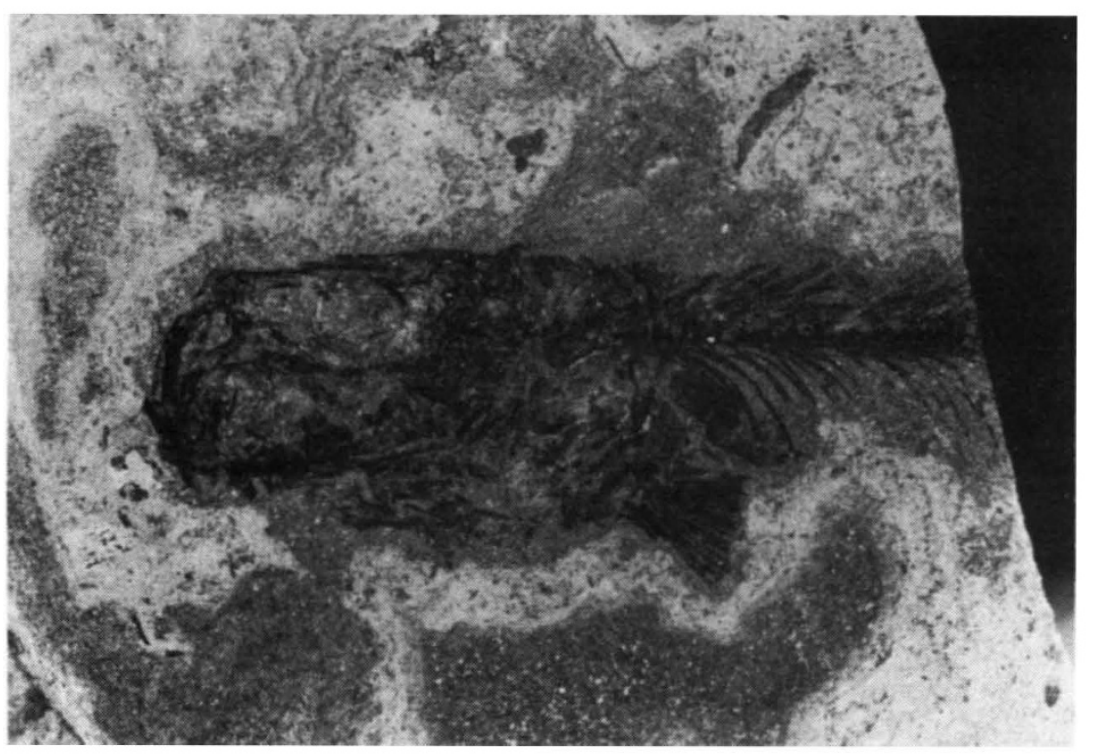

